University of Nebraska - Lincoln

DigitalCommons@University of Nebraska - Lincoln

Faculty Publications from the Harold W. Manter Laboratory of Parasitology

2001

\title{
Evaluation of the Specificity of Five Oligoprobes for Identification of Cyathostomin Species from Horses
}

\author{
J. E. Hodgkinson \\ University of Liverpool
}

S. Love

University of Glasgow

J. Ralph Lichtenfels

Animal Parasitic Disease Lab, ARS, United States Department of Agriculture, 2jrcgl@gmail.com

S. Palfreman

University of Liverpool

Y. H. Ramsey

University of Glasgow

See next page for additional authors

Follow this and additional works at: https://digitalcommons.unl.edu/parasitologyfacpubs

Part of the Parasitology Commons

Hodgkinson, J. E.; Love, S.; Lichtenfels, J. Ralph; Palfreman, S.; Ramsey, Y. H.; and Matthews, J. B., "Evaluation of the Specificity of Five Oligoprobes for Identification of Cyathostomin Species from Horses" (2001). Faculty Publications from the Harold W. Manter Laboratory of Parasitology. 631.

https://digitalcommons.unl.edu/parasitologyfacpubs/631

This Article is brought to you for free and open access by the Parasitology, Harold W. Manter Laboratory of at DigitalCommons@University of Nebraska - Lincoln. It has been accepted for inclusion in Faculty Publications from the Harold W. Manter Laboratory of Parasitology by an authorized administrator of DigitalCommons@University of Nebraska - Lincoln. 


\section{Authors}

J. E. Hodgkinson, S. Love, J. Ralph Lichtenfels, S. Palfreman, Y. H. Ramsey, and J. B. Matthews 


\title{
Evaluation of the specificity of five oligoprobes for identification of cyathostomin species from horses
}

\author{
J.E. Hodgkinson ${ }^{\mathrm{a}, *}$, S. Love ${ }^{\mathrm{b}}$, J.R. Lichtenfels ${ }^{\mathrm{c}}$, S. Palfreman ${ }^{\mathrm{a}}$, Y.H. Ramsey ${ }^{\mathrm{b}}$, J.B. Matthews ${ }^{\mathrm{a}}$ \\ ${ }^{a}$ Division of Equine Studies, Department of Veterinary Clinical Sciences and Animal Husbandry, University of Liverpool, Leahurst, \\ South Wirral, CH64 7TE, UK \\ ${ }^{\mathrm{b}}$ Division of Equine Studies, Department of Veterinary Clinical Studies, University of Glasgow, Bearsden Road, Glasgow, G61 1QH, UK \\ ${ }^{\mathrm{c}}$ Biosystems and National Parasite Collection Unit, Agricultural Research Service, US Department of Agriculture, Beltsville, MD 20705, USA
}

Received 19 September 2000; received in revised form 27 November 2000; accepted 27 November 2000

\begin{abstract}
Here, we report evaluation of five oligoprobes designed from intergenic spacer (IGS) region sequences for identification of cyathostomin species. Oligoprobes were designed for identification of Cylicocyclus ashworthi, Cylicocyclus nassatus, Cylicostephanus longibursatus, Cylicostephanus goldi and a fifth probe designed to identify all members of this tribe. PCR amplification of IGS DNA from 16 cyathostomin species allowed sequence comparison and identification of four putative species-specific probes. Southern blotting of amplified products from 16 species showed that all probes were species-specific. The fifth probe recognised all 16 cyathostomin species but did not bind to members of the genus Strongylus. Furthermore, these probes were used to identify individual infective L3, eggs and L4 indicating that they will be invaluable to furthering the study of the epidemiology and pathogenesis of these important equine nematodes. (C) 2001 Australian Society for Parasitology Inc. Published by Elsevier Science Ltd. All rights reserved.
\end{abstract}

Keywords: Cyathostominae; Horses; Intergenic spacer region; Nematoda; Species-specific DNA probes

Nematodes of the subfamily Cyathostominae (cyathostomins) are the most common parasites of horses (Langrova, 1998). Infections with these parasites are complex and more than 50 species have been documented (Lichtenfels et al., 1998), however, most horses carry a burden of five to ten common species (Reinemyer et al., 1986; Torbert et al., 1986; Bucknell et al., 1995). Clinically, these parasites are associated with various syndromes including larval cyathostominosis, a fatal enteritis that occurs secondary to synchronised reactivation of inhibited larvae from the intestinal mucosa (Giles et al., 1985; Vanloon et al., 1995). This syndrome has a $50 \%$ case fatality rate even when intensive treatment is instituted (Love and McKeand, 1997). More subtly, these nematodes induce a protein-losing enteropathy, resulting in loss of condition and peripheral oedema (Love et al., 1992a,b; Murphy and Love, 1997) or mild weight loss, poor appetite and lethargy with disordered intestinal motility (Matthews and Morris, 1995). Unfortunately, nothing is known of the association between the individual cyathostomin species and the development of

\footnotetext{
* Corresponding author. Tel.: +44-151-794-6010; fax: +44-151-7946034.

E-mail address: jhodgkin@liverpool.ac.uk (J.E. Hodgkinson).
}

these different clinical syndromes. Due to the potential number of species involved it is difficult to establish a means of diagnosis and a therapeutic plan to deal with these nematodes (Klei and French, 1998). This is compounded by the fact that there is a relative lack of immunity, so that animals can act as sources of pasture contamination throughout their lives (Beelitz and Gothe, 1997). Excessive use of anthelmintics, particularly benzimidazoles, has led to development of resistance. To understand the epidemiology and pathogenesis of cyathostomins in more detail, it is necessary to approach them on a single species basis. The current method of identifying cyathostomin species is to remove the adult worms at necropsy and examine their morphology, which is of little value in clinical veterinary practice. Furthermore, species identification of eggs is not possible, and the identification of larvae is difficult and time-consuming using morphological parameters. Previously, we published the sequence of part of the intergenic spacer (IGS) region of the ribosomal DNA of 16 cyathostomin species: Cylicocyclus ashworthi, Cylicocyclus auriculatus, Cylicocyclus radiatus, Cylicocyclus insigne, Cylicocyclus leptostomum and Cylicocyclus nassatus; Cylicostephanus bidentatus, Cylicostephanus calicatus, Cylicostephanus goldi, Cylicostephanus longibursatus and 
Cylicostephanus minutus; Cyathostomum pateratum, Cyathostomum catinatum, Coronocyclus coronatus and Coronocyclus labiatus and Tridentoinfundibulum gobi (Kaye et al., 1998). Sequences across this region showed intraspecific variation from 0.2 to $2.2 \%$, with an interspecific variation between 3 and $60 \%$, sufficient difference to indicate that this region may be useful for species differentiation. Based on these nucleotide differences, we have designed oligonucleotide probes for identification of four common species. These species were selected on the basis of their high prevalence and/or high intensity of infection (Ogbourne, 1976; Lyons et al., 1997). Here, we have used a non-radioactive digoxigenin (DIG) hybridisation system to validate the species-specificity of these four oligonucleotide probes.

Individual adult worms were collected from the dorsal colon, ventral colon and caecum of horses at a local abattoir.

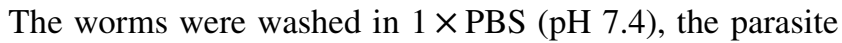
heads removed for morphological identification and the remainder of the parasites stored in liquid nitrogen. Nematode heads, usually including the entire oesophagus, were cleared in phenol-alcohol $(80 \%$ melted phenol crystals and $20 \%$ absolute ethanol) in temporary wet mounts on microscope slides and identified with the aid of interference light microscopy at $400 \times$ magnification. Identification to genus and species followed the recommendations of recent international workshops outlined by Lichtenfels et al. (1998). Strongyle eggs were collected from positive faecal samples by flotation in saturated salt solution, washed in de-ionised water, and stored at $-20^{\circ} \mathrm{C}$. Subsequently, individual eggs were harvested at $40 \times$ magnification and placed singly into $500 \mu \mathrm{l}$ tubes for use in the PCR. Cyathostomin L3 were collected following harvesting from pasture samples (Jorgensen, 1975) and L3 selected in a similar manner to the eggs and stored at $4{ }^{\circ} \mathrm{C}$. Prior to use in PCR amplification, L3 were exsheathed in $0.1 \%$ sodium hypochlorite w/v/ $0.825 \% \mathrm{NaCl} \mathrm{w} / \mathrm{v}$ in $\mathrm{PBS}$, for $5 \mathrm{~min}$ at room temperature and then washed extensively in PBS. Cyathostomin L4 were harvested by hand from faeces of clinical cases, identified as cyathostomins under a stereomicroscope washed in PBS, and stored at $-70^{\circ} \mathrm{C}$ until used individually in the PCR. DNA was extracted from three individual adult worms of each species, with the exception of the following rarer species within our population, Cylicocyclus ultrajectinus, Poteriostomum imparidentatum (two individuals) and Coronocyclus coronatus (one individual). Worms were digested at $55^{\circ} \mathrm{C}$ overnight in $200 \mu$ of extraction buffer containing $200 \mathrm{mM}$ Tris-HCl (pH 8.0), 50 mM-EDTA, 200 $\mathrm{mM} \mathrm{NaCl}, 1 \% \mathrm{SDS}$ and $20 \mathrm{mg} / \mathrm{ml}$ Proteinase $\mathrm{K}$. The DNA was purified by two phenol/chloroform extractions, precipitated with ethanol, dissolved in deionised water and stored at $4^{\circ} \mathrm{C}$. In the case of L4 stages, genomic DNA was extracted from individual larvae exactly as described for the adult worms. In addition, genomic DNA was extracted from individuals of Parascaris equorum (one individual), Oxyuris equi (one individual) and Strongylus edentatus (nine individuals) as described for the cyathostomins. Equine genomic DNA was extracted from blood using the DNace Megablood kit (Bioline, UK.) as per manufacturer's instructions.

PCR reactions were performed in $50 \mu \mathrm{l}$ volumes using primers (CY1, forward; 5'-GGTCAAGGTGTTGTATCCAGTAGAG-3' and CY18, reverse; 5'-CTTAGACATGCATGGCTTAATC- $3^{\prime}$ ) that amplify the IGS region of all 16 cyathostomin species studied here (Kaye et al., 1998). Amplification reactions contained $50 \mathrm{mM} \mathrm{KCl}, 10 \mathrm{mM}$ Tris- $\mathrm{HCl}$ ( $\mathrm{pH} 8.3$ ), $1.5 \mathrm{mM} \mathrm{MgCl}_{2}, 250 \mu \mathrm{M}$ of each dNTP, 1.25 U Amplitaq Gold (PE Biosystems) and between 0.02 and 0.1 volumes of template genomic DNA. In some cases, the primer CY 26 (5'-GAGCTGGGTTTAGACCGTCGTAG-3') was used in combination with CY 18. This primer was designed over a more conserved region of the 26s rDNA and was used to confirm integrity of DNA from the more distantly related nematode species, $P$ equorum and O. equi. In the case of eggs and L3, genomic DNA was not extracted and whole parasites were subjected to three cycles of freeze/boiling prior to addition to the PCR reaction. The cycling conditions were as follows: denaturation/Amplitaq Gold activation for $10 \mathrm{~min}$ at $94^{\circ} \mathrm{C}$, denaturation at $94^{\circ} \mathrm{C}$ for $1 \mathrm{~min}$, annealing at $60^{\circ} \mathrm{C}$ for $1 \mathrm{~min}$ and extension at $72^{\circ} \mathrm{C}$ for $2 \mathrm{~min}$ for 35 cycles, followed by a final extension at $72^{\circ} \mathrm{C}$ for $10 \mathrm{~min}$. Control samples were included where template DNA was absent. As controls for non-specific amplification, reactions were prepared that included $100 \mathrm{ng}$ of equine genomic DNA or $5 \mu \mathrm{l}$ of equine cyathostomin-negative faeces, all performed in triplicate. Integrity of the equine DNA was determined by PCR with primers for three MHC class 2 loci, DQA (forward $5^{\prime}$ CTGAICACITTGCCTCCTATG-3' and reverse $5^{\prime}$-TGGTAGCAGCAGIAGIGTTG-3'), DRA (forward 5'-GCTTCTCATCCTAGTTCCCTT and reverse GCCTAGGAGTGCAGCAGA-3'), DQB (forward 5' CTCGGATCCGCATGTGCTACTTCACCAACG-3' and reverse $5^{\prime}-\mathrm{GAG}-$ CTGCAGGTAGTTGTGTCTGCACAC- $3^{\prime}$ ) C. catinatum PCR products were gel purified using Spin-X columns (Corning, USA) as per manufacturer's instructions and cloned into pCR2.1 vector (TA-cloning kit, Invitrogen). Sequencing was performed on an ABI automated sequencer using T7 (forward) and M13 (reverse) primers (MWGBiotech, Germany).

The alignment of IGS sequences for 14 cyathostomin species is shown in Fig. 1, along with the sequences of the oligoprobes used here. Each probe was selected on the basis of low sequence identity across this region with that of the other 13 species. Particular attention was paid to compare those species which showed highest sequence similarity across the entire IGS region. Once designed, the oligonucleotide sequences were tested by alignment (FASTA) analysis against all sequences in the database, using GCG Wisconsin. A high level of conservation of sequence downstream of the region shown in Fig. 1 enabled design of a probe common to all cyathostomin species. This was desig- 


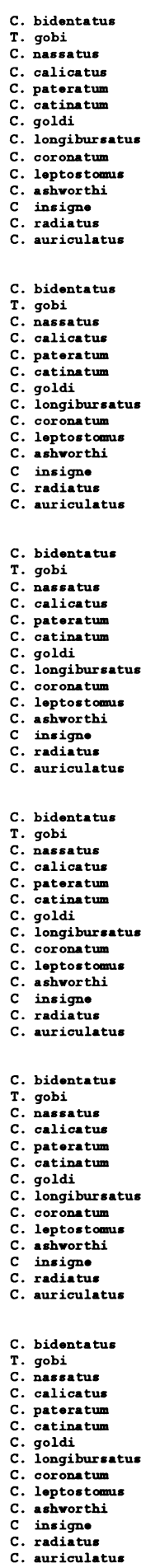

5. TTGTCTTCTG CTGTCTAGgG GGTGTTTTAA ATCACCTCCT AGCGCTGAG. GAAAAATACA GCTAAGTCTA GTGACTAGGT GTA.RATTCT ATAGGTCTTA CATGGGATTT GTATTTGTGT TIGTCTICTA CTGTCTAGGG GGTGTTTTAA TTCACTCTCT AGCGTGATCA GGAAAAAT.A GCTAAGTGCG GTGAATAAGT GC.TCATICT GGTCCACAGT AACATTEATT ATAATTTATG TTGTCTTCTC CTGTCTAGGG GGTGTTTTAA ATCACTTCTT AGOGTTGGGA GAAA. .TTTA GCCAAGTGTG GCGACTAGGC GTA.CATCAT A. COGTCCCA CATAGAAAAT GTATTTGCTT TTGTCTTCTC CTGTCTAGGG GGTGTTTTAA ATCACTTCTT AGCGTTGGGA GAAA. .TTTA GCCAAGTGTG G. GACTAGGC GTA. TATCAT A. OGGTCCC. CATAGAAAAT GTATTTGCTT TIGTCTTCTC CTGTCTAGGG GGTGITTTAA ATCACTTCTT AGCGTTGGGA GAAA. .TTTA GCCAAGTGTG GCGACTAOGC GTA. CATCAT A. CGGTCCTA CATAGAAAAT GTATTTGCTT TTGTCTTCTC CTGTCTAGGG GGTGTTTTAA ATCACTTCTT AGCATC, GEA G.M. . TITA GCCAA. .GTA ACGACTAGCC GTA.AATCAT A.TGGTCCTA CATAG.AAAT GTACTTGCTT

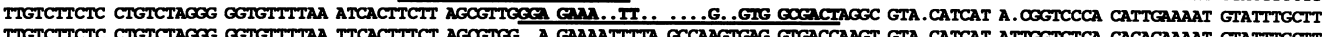
TTGTCTTCTC CTGTCTAGGG GGTGTTTTAA TTCACTTTCT AGOGTGG. . G GAAATTTTA GCCAAGTGAG GTGACCAAGT GTA. CATCAT ATTEGTCTCA CACAGAAAAT GTATTTGCTT TIGTCTTCTC CTGTCTAGG GGTGTTTTAA TTCACTTTCT AGCGTGG. .G GAAAACTTIA GCCAAGTGTG GTGACCAAGT GTA.CATCAT ATTGGTCTCA CATAGAAAT GTATTTGCTT

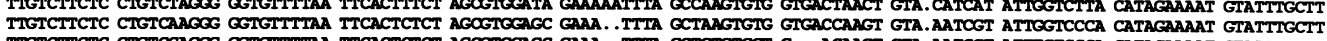
TTGTCTTCTC CTGTCCAGGG GGTGTTTTAA TTCACTCTCT AGCGTGCAGC GAAA. .TTTA GCTGTGTGGT G. ..ACAAGT GTA.AATCGT ATTGGTCCCA CATACAAAAT GTATTCA CATAGAAAT GTATTTCCAT TTGTCTTCTC CTGTCCAGGG GGTGTTTTTT TTCACTCTCT AGCGCGCAGC GAAA..TTTA GCCAAGTGTG GTEACTAAGT GTA.CATCGC ATTGGTCCTA CATAGAAAAT GTATTTG.TT

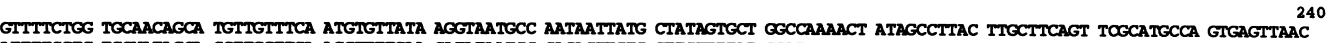

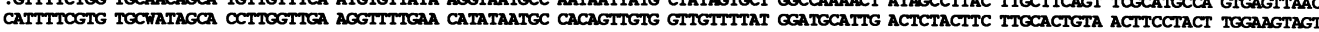
CAGTGTAACG TGGG.. TCGG TTTGGGTAAA AATEACTTAT ACAGTGCTGG TCAAAACTTT AGCCTAAGCC TTGGGCTTAG GCTTAGGCTT AGGCTTAGGC TIAC...........GGAAAA CGTTCTGAAT AGCT..AG.. . ....TTTTCA ATGTTGCGGT CACATAATGC TATTGA. .GT ATEGCATTCT TTTATGAAAG CACT. .GTTT TCACTTAATA CAAT.......... TGTAAC CGTTCTGAAT AGCT..AG.. .....TTTTCA ATGTTGCAGT CACATAATGC TATTGA. .GT ATEGCATTCT CTTATGAAAG CACT..GYTT TCACTTCATA CAGT.......... TGTAAC

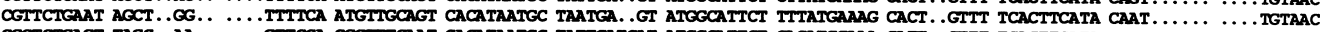

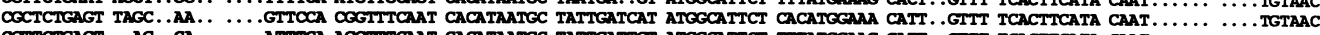
OGITCTGAGT ..AG.. CA.. ....ATTTCA AGGTTTCAAT CACATAATGC TATTGATTGT ATGGCATTCT TrTATGEAAG CATT. .GTTT TCACTTCATA CAAT........... TGTAGC CATTT. ... TGGT. .GGOC TCTAGITTCA AGGTTTTAAT CGCATAATGC TGACATATGT ATGCCATTCT TTTATACAAG CATT. .AATT CTACTTCATG CAGT...........GTAAAA

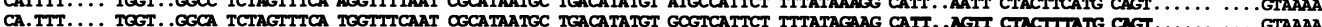
CGetroeg tegr CGTTTTCGA TEGT..GACA TCTAGTTTCA AGGATTCACT CACATATTGA CAACAAACGT ATGTCAATCT TrCATAGAAT CATT..GATT CTACATATAC AATG.......... TAACAT GTTTCEGTG GTEA.. CATC TCTAATTTCA AGGATTCACT CACATTTTGC TAACAAACGT ATGTCAATCT TTTAAAGAT TATT..GATt CTA. TTATAC AATG............TAACAT

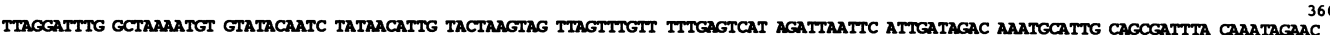
GACTTATAAT TEAATAAATG TAGTTGATGT TCTATGCTTT CCOCACATTT ATGTACGAAA CATCATTTTG AAATAATTTC CAACGTTAAA ATGTGCTCTT TATACAATTT CAAAATGTGC GCCACACTGT GATTTTGCTA TATCTTACTC AGTTTCGAAA RACAGAAACC AAATTCOCAT ATTGCGTGAA TCACACTGCT TAGCGTTTAG TCAACAAATA CTAAAGTTTC ATTACACTTC ATTCTCATTT GGTATAAAOG GCTTACAACT CTGTATATGT AAGTGTAATC AGTGTTTCAA CTTT. TATCA GCACTTCTAT GTGCCAAACA CTGAGAATA ATGTGCTTAT GTGAATGTT ATTCTOGTAT GGTATAAATG @CTTACACCT CTGTATATGT AAGTGTAATC AGTGTTTCAA CTTTATATCA GCACTTCTAT GTGCCAAACA CTGACAAATA ATGTGCTTAT GTGAATGTTI ATTTOGTTT GGTATAAATG GCTTACAACT CTGTATATGT AAGTGTAATC AGTGTTTCAA CTTTGTATCA GCACTTCTAT GTGCCAAACA CTGCGAAATA ATGTGCTTAT GTGAATGTTT A......... .GTATAAATG GCTTACAACT CTGTATATGT AAATGTAATC AATGTTTAAA CTTTATCTCA GCACTTCGAT GTGCCAAACA CTGAAAAATA TTATGCTTAT GTGAATGTTT ATCCATT GGTATAAATG GCTTACAACT TTGTATATGT AAGTGTAATC AATGTTTCAA CTTTATATCA GCACTTCEAT GTGCCAAACA CTGAGAAAT ATGCGCTTGT GTGAGTGTTT

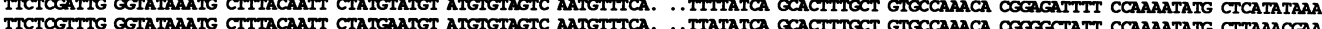

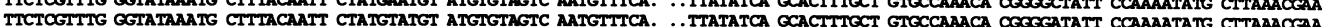

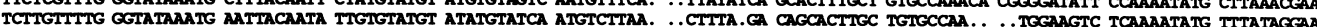

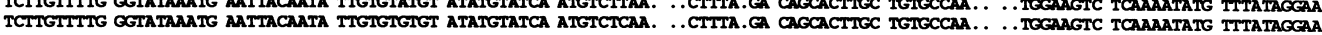
TCTTGITTTG AGTATGAATG AATCACAGIT TTATGCATGT ATGTGTATCA GTGTCTCAA. ...CTTTAGGT CAGCACTTGC TGTGCCAAAC ACTGGAaGTT TTGAAATATG TTTATATGA

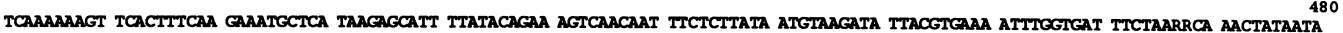

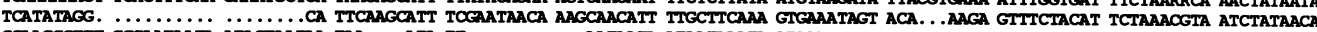
ceaAgectTt ggeataAta a.......

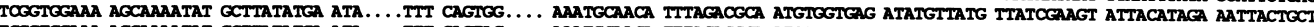
TOGGTGGAAA AGCAAAATAT GCTTATATEA ATA.... TTT CAGTAG.... AAATECAACA TTTACACGCA ATGTGGTGAG ATATGTTATG TTATCGAAG ATTACATAGA AATTACTGCT

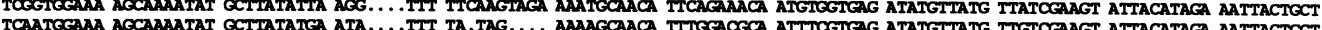
TCAATGGAAA ACCAAMATA

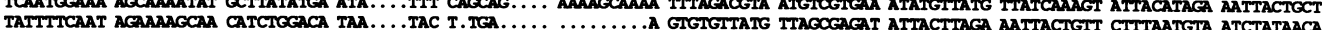
TATTTIC

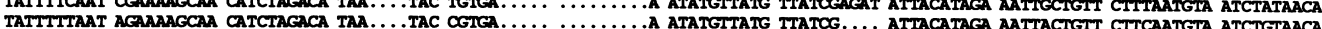

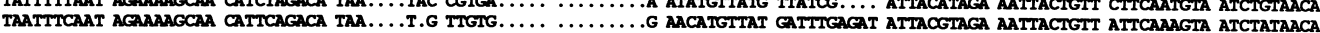

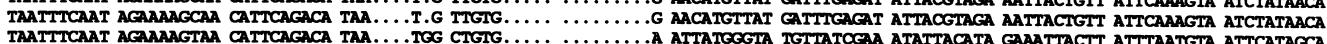

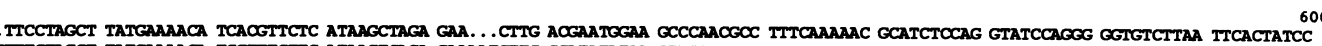
TTTTCTAGCT TATEAAAACA TCGTTTCTTC ATAAGTCACA GAAAATGTTG CGATATATAA GTYTTAACGTC TCTAAACAAT GCATTTCTTG GCRTCTAGGG GGTGTCTTAA TTCACT. GAC TTEAAATGTT TTCCACATAT G. .AAACAAC TTTGEAAATT GCAACAACTT CGCTGAAATG OCTGTAATCA TGATGTTCAG GGGGTGTTTT AATTCACTAG CAATGCAGAC AGGGGGTGTT CACG CACCETACA ATCTATAM I

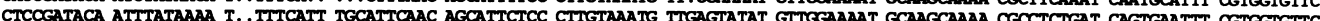
TTTCGTACA .. TATAACA T. TTTC C CTTCAAC AGCATTCTCC CTTGTAAATG TTGAGTATAT GTTGGAAAA GCAAGCAAAA CGCCTCTGAT CAGTGAATTT CGTGGTGTTC TTCTEATTTG AaAAC.ATT T. TTTCCAC A.CTATCAAC AGCATTTTCC CTTGTAAATG TT. CGAGCAT GTTGAAAAAT GCAAGCAAAA CGOCTCTGAT CAATGCATTT CGTGGTGTTC

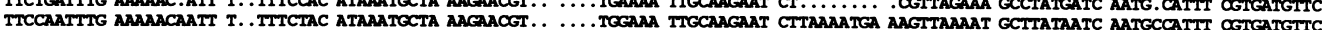

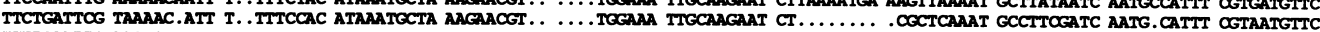

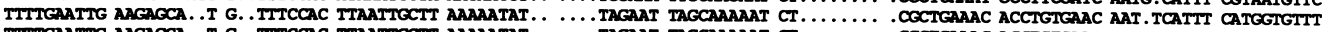

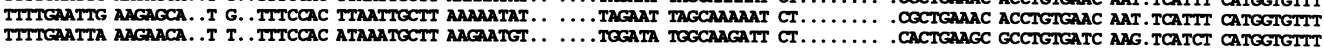

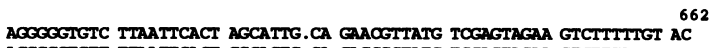
AGGGGGTGT TTAATTCACT GGCAGTG.CA GAGOGCTATG TCGAGTAGAA GTCTTTTTGT AC TTAATTCACT AGCAATG.CA GACCGTTATG GTGCATTTCG TCGAGTAGAA GTCTTITIGT AC AOGGGTGTT TIAATTCACC AGCATTG.CA TATCGTTATG TCGAGTAGAA GTCTTTTTTGT AC AGGGGGTTT TTAATTCACT AGCATTG.CA TATCGTTATG TCAGGTAGAA GTCTTTTTGT AC AGGGGTGTT TTAATTCACT AGCATTG.CA TATCGTTATG TCGAGTAGAA GTCTTTTIGT AC AGGGGGTTT TIAATTCACT AGCATTG.CA TATCGTTATG TCEAGTAGAA GTCTTITIGT AC AOGGGETGTT TTAATTCACT AGCATTG.CA GACOGTTATG TCEAGTACAA GTCTTTTIGT AC AOGGGGTGTT TTAATTCACT AGCATTG.CA GACOGTTATG TCEAGTACAA GTCTTTTTGT AC AGGGGGTTT TTAATTCACT AGCATTG.CA GACOGTTATG TCGAGTACAA GTCTTTTTGT AC AGGGGGTGT TTAATTCACT TGCATTG.CA GACOGTTATG TCEAGTACAA GTCTTITTGT AC AGGEAGTGTT TTAATTCACT TGCATTG.CA GAGCGTTATG TCGAGTAGAA GTCTTTTTGT AC

Fig. 1. Partial sequence of the IGS from 14 cyathostomin species showing multiple alignment of the variable region. Dots represent gaps inserted to optimise alignment. The four short species-specific sequences used as probes in Southern blotting are shown in bold and underlined. The region downstream of this sequence is not shown but includes approximately $430 \mathrm{bp}$ of sequence which is highly conserved in all 14 species. The Pan probe was designed from this region.

nated the 'Pan' probe (5'-CGGCGAATTTGCGGTTGCCTTCGCGTT-3'). DIG-labelled oligonucleotide probes were prepared by tailing using terminal transferase
(Roche, DIG Oligonucleotide Tailing Kit) using 100 pmoles of oligonucleotide per reaction. Southern blotting was performed using the Pan probe and species-specific probes. 
Amplification products $(8 \mu \mathrm{l})$ and $10 \mu \mathrm{l}$ of DIG DNA ladder (Roche) were visualised on ethidium bromide stained $1 \%$ agarose gels by UV transillumination. Southern blotting, using the non-radioactive DIG-labelling system (Roche), was carried out following electrophoresis. Gels were denatured for $15 \mathrm{~min}$ at room temperature in $0.5 \mathrm{~N} \mathrm{NaOH}, 1.5 \mathrm{M}$ $\mathrm{NaCl}$ and rinsed in sterile water, this was followed by two

A

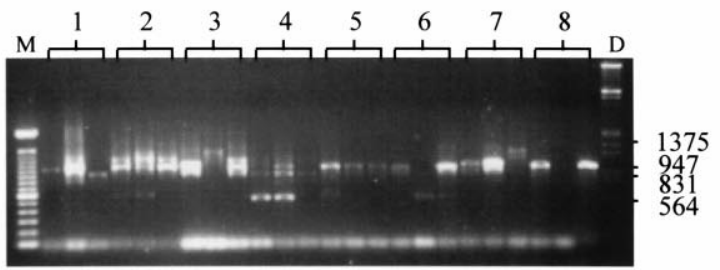

$\begin{array}{llllllllll}9 & 10 & 11 & 12 & 13 & 14 & 15 & 16 & 17 & 1819\end{array}$

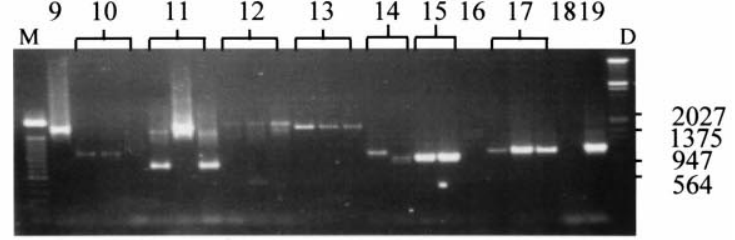

B
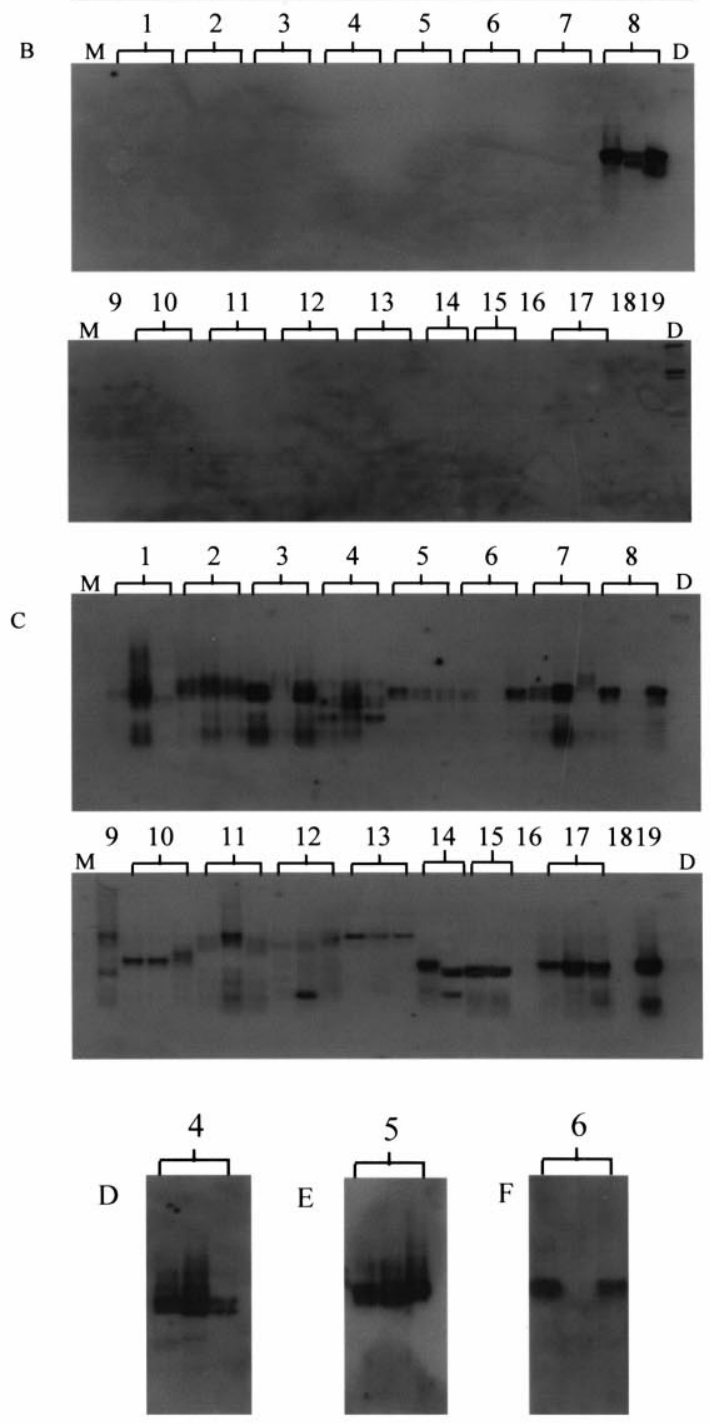

10 min washes at room temperature in neutralisation solu-

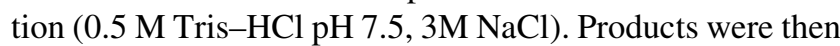
transferred onto nylon membrane (Osmonics, GRI) by capillary blotting overnight in $20 \times$ standard saline citrate (SSC) and fixed by UV cross-linking. Membranes were prehybridised in $15 \mathrm{ml}$ of standard hybridisation buffer $(5 \times \mathrm{SSC}, 0.1 \% \mathrm{~N}$-lauryolsarcosine, $0.02 \%$ SDS, $1 \%$ blocking reagent (Roche), $0.1 \mathrm{mg} / \mathrm{ml}$ poly A) at $32^{\circ} \mathrm{C}$ for $2 \mathrm{~h}$. Hybridisation was carried out at $32^{\circ} \mathrm{C}$ for $2.5 \mathrm{~h}$ in fresh standard hybridisation solution containing $0.1-2 \mathrm{pmol} / \mathrm{ml}$ of probe. Membranes were washed twice at room temperature in $2 \times \mathrm{SSC}, 0.1 \%$ SDS for $5 \mathrm{~min}$ each time and twice for $15 \mathrm{~min}$ at $32^{\circ} \mathrm{C}$ in $0.5 \times \mathrm{SSC}, 0.1 \%$ sodium dodecyl sulfate (SDS). Membranes hybridised with the Pan probe or the probe for $C$. nassatus were washed twice at a higher stringency $(0.1 \times \mathrm{SSC}, 0.1 \% \mathrm{SDS})$, at $32^{\circ} \mathrm{C}$ for $15 \mathrm{~min}$. For detection of hybridised probe, membranes were prewashed for $2 \mathrm{~min}$ in DIG wash buffer (Roche, 1\% blocking solution, $0.3 \%$ Tween 20), then incubated for $30 \mathrm{~min}$ in $1 \%$ blocking solution. The membranes were placed into blocking solution containing a dilution of 1:10 000 anti-DIG-alkaline phosphatase (Roche) and incubated for a further $30 \mathrm{~min}$. Next, the membranes were washed twice for $15 \mathrm{~min}$ in DIG washing buffer, equilibrated in detection buffer $(100 \mathrm{mM}$ Tris$\mathrm{HCl}, \mathrm{pH} 9.5,100 \mathrm{mM} \mathrm{NaCl}$ ) and briefly blotted onto $3 \mathrm{MM}$ paper (Whatman), covered in the chemiluminescent substrate CSPD (Roche) and incubated between two sheets of acetate for $5 \mathrm{~min}$. Finally, the membranes were incubated at $37^{\circ} \mathrm{C}$ for $10 \mathrm{~min}$ and exposed to autoradiographic film between $5 \mathrm{~min}$ and $12 \mathrm{~h}$.

PCR products were amplified from genomic DNA of adult worms from 16 different cyathostomin species using primers CY1 and CY18 (Fig. 2A). These different species of adult worms were isolated from several horses at two geographical locations (Table 1). In the case of the cyathostomin species, a single product of $1 \mathrm{~kb}$ in size was seen upon electrophoresis (Fig. 2A). Although this product was predo-

Fig. 2. (A) Electrophoresis in a $1 \%$ agarose gel of the amplification products from 16 species of cyathostomin using primers CY1 and CY18. (B) Southern blot of gel in 2A hybridised with 19 mer DIG-labelled C. longibursatus probe. (C) Southern blot of gel in 2A hybridised with 27 mer DIG-labelled Pan probe. Lanes: M, 100bp ladder, group 1, three $C$. calicatus individuals, group 2, three $C$. pateratum individuals, group 3 , three $C$. catinatum individuals, group 4 , three $C$. nassatus individuals, group 5, three $C$. goldi individuals, group 6, three $C$. ashworthi individuals, group 7 , three $C$. bidentatus individuals, group 8 , three $C$. longibursatus individuals, group 9 , one $C$. coronatus individual, group 10 , three $C$. leptostomum individuals, group 11 , three $C$. labratus individuals, group 12 , three $C$. minutus individuals, group 13, three C. labiatus individuals, group 14, two C. ultrajecti$n u s$ individuals, group 15 , two $P$. imparidentatum individuals, group 16 , one $S$. edentatus individual, group 17, three $C$. insigne individuals, group 18, PCR negative control, group 19, PCR positive control, D, DIG-labelled molecular weight marker. (D) Southern blot showing hybridisation of $C$. nassatus probe against individuals of homologous species, lack of hybridisation to other 15 species not shown. (E) As D except hybridisation was with $C$. goldi probe. (F) As D except hybridisation was with $C$. ashworthi probe. 
minant, some species (particularly C. catinatum, C. pateratum and $C$. nassatus) consistently generated more than one product, with up to three bands from 0.7 to $1.2 \mathrm{~kb}$ in size detected (Fig. 2A, group 2, group 3 and group 4). To compare the sequence of these other bands, the three products amplified from C. catinatum DNA (Fig. 2A, group 3) were purified, cloned and sequenced. These products were shown to contain regions of DNA of two or three tandem repeats of 184 nucleotides, which accounted for the difference in size of the larger products (Fig. 3). In some cases a small band of 500 bp was amplified (Fig. 2A, group 2, group 4 and group 6). For C. coronatus, C. labiatus and $C$. minutus, single bands of $1.7 \mathrm{~kb}, 2 \mathrm{~kb}$ and $2.1 \mathrm{~kb}$ were amplified, respectively. As reported previously these different sized products are due to an extended region of IGS sequence at the $3^{\prime}$ end near the $18 \mathrm{~S}$ rRNA gene for $C$. coronatus, whilst $C$. labiatus and C. minutus have an extended internal region (Kaye et al., 1998). C. labratus, whose sequence across this region of the IGS has not been previously reported, also amplified a larger product of 1.7 kb (Fig. 2A, group 11). From S. edentatus individuals, a single product of $1.5 \mathrm{~kb}$ was amplified (Fig. 4) and this was consistent amongst individuals. No amplification products were detected when the CY1 and CY18 primers were used in reactions with DNA extracted from $O$. equi and $P$. equorum individuals (Fig. 4). Integrity of DNA from these species was confirmed by amplification of IGS region using highly conserved primers, CY18 and CY26 (Fig. 4). When used in the PCR reaction, equine DNA and faecal material gave no amplification products using CY1 and

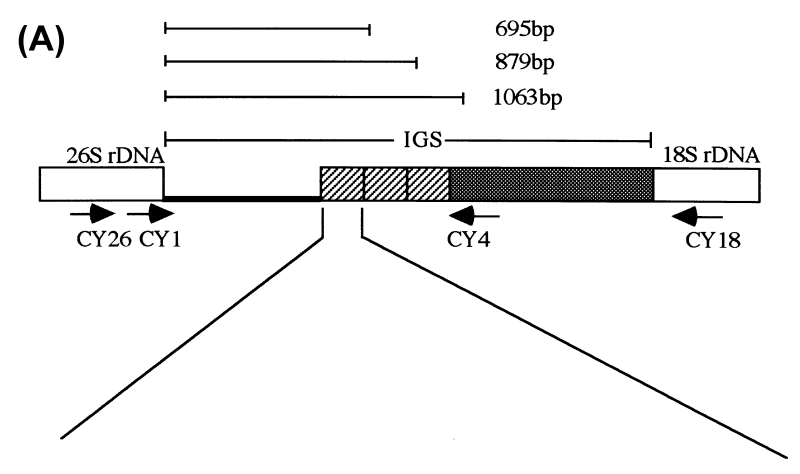

(B) $\begin{array}{rlll}\text { IGS repeat } 5 \text { ' } & \text { GTGAGATATG TTATGTTATC GAAGTATTAC ATAGAAATTA } & 40 \\ \text { CTGCTCACCG ATACAATCTA TAACATTTTC ACTTTAACAG } & 80 \\ \text { CATTTTCCCT TGTAAATGTT CGAAAATGTT GGAAAATGCA } & 120 \\ \text { AGCAAAACGC TTCAAATCAA TGCATTTCGT GGTGTTCAGG } & 160\end{array}$ $\begin{array}{lll}\text { AGCAAAACGC TTCAAATCAA TGCATTTCGT GGTGTTCAGG } & 160 \\ \text { GGGTGTTTTA AATCACTAGC ATTG } & 3^{\prime} & 184\end{array}$

Fig. 3. (A) Schematic of C. catinatum rDNA showing location of IGS repeat region. Unfilled boxes represent $99 \%$ conserved rDNA sequence, hatched box 95-98\% conserved IGS sequence, the thick black line represents the variable region and the striped box represents three $100 \%$ identical $184 \mathrm{bp}$ IGS repeat region found in C. catinatum individuals. Arrows indicate position of PCR primers. One, two or three copies of the $184 \mathrm{bp}$ repeat are found in the 695,879 and 1063 bp amplified products, respectively, when PCR was carried out using primers CY1 and CY4 (CGGTACAAAAAGACTTCTACTCG). (B) DNA sequence of the 184 bp IGS repeat region.

CY18 primers (not shown). Integrity of equine DNA was confirmed by amplification of bands from the equine MHC region using equine specific primers designed from DRA, DQA and DQB sequences (not shown).

Table 1

Cyathostomin species evaluated in this study ${ }^{\mathrm{a}}$

\begin{tabular}{|c|c|c|c|}
\hline Species studied & $\begin{array}{l}\text { Number of individual } \\
\text { worms examined }\end{array}$ & Geographical location & Number of horses \\
\hline \multicolumn{4}{|l|}{ Cyathostomum } \\
\hline catinatum & 3 & 2 Scotland, 1 England & 2 \\
\hline pateratum & 3 & Scotland & 1 \\
\hline \multicolumn{4}{|l|}{ Coronocyclus } \\
\hline coronatus & 1 & Scotland & 1 \\
\hline labiatus & 3 & England & 1 \\
\hline labratus & 3 & England & 1 \\
\hline \multicolumn{4}{|l|}{ Cylicostephanus } \\
\hline calicatus & 3 & Scotland & 1 \\
\hline minutus & 3 & England & 1 \\
\hline longibursatus & 12 & 2 Scotland, 10 England & 3 \\
\hline goldi & 12 & 3 Scotland, 9 England & 2 \\
\hline bidentatus & 3 & Scotland & 1 \\
\hline \multicolumn{4}{|l|}{ Cylicocyclus } \\
\hline ashworthi & 13 & 3 Scotland, 10 England & 3 \\
\hline insigne & 3 & England & 1 \\
\hline leptostomum & 3 & England & 1 \\
\hline nassatus & 13 & 3 Scotland, 10 England & 4 \\
\hline ultrajectinus & 2 & Scotland & 1 \\
\hline \multicolumn{4}{|l|}{ Poteriostomum } \\
\hline imparidentatum & 2 & Scotland & 1 \\
\hline
\end{tabular}

${ }^{a}$ This table shows the number of individuals from each species which have been used in the evaluation of probe specificity using the Southern blot technique. Geographical location of isolates is indicated. Number of horses refers to the total number of horses from which the individuals of each species were isolated. 


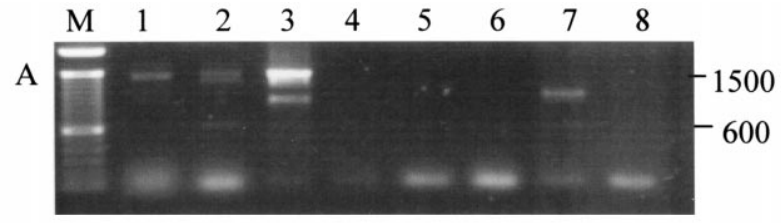

$\begin{array}{llllllllllllllll}\mathrm{M} & 1 & 2 & 3 & 4 & 5 & 6 & 7 & 8 & 9 & 10 & 11 & 12 & 13 & \mathrm{D}\end{array}$

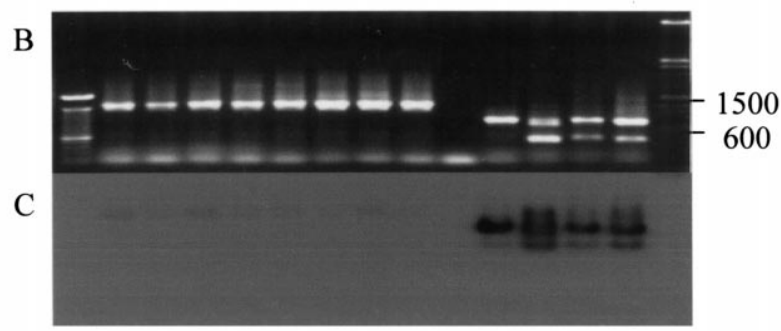

Fig. 4. (A) Electrophoresis in a $1 \%$ agarose gel showing the results of amplification from $P$. equorum and $O$. equi using primers CY26 and $\mathrm{CY}$ 18 (lanes 1-3) and primers CY1 and CY18 (lanes 5-7). Lanes: M, 100 bp ladder; 1 and 5, P. equorum individual; 2 and 6, O. equi individual; 3 and 7 , positive PCR control using adult cyathostomin template; 4 , PCR negative control for CY 26 and CY 18 primers; 8, PCR negative control for CY1 and CY 18 primers. (B) Electrophoresis in a $1 \%$ agarose gel of the amplification products of eight $S$. edentatus individuals using primers CY1 and CY 18 (lanes 1-8). (C) Southern blot of gel in B probed with the Pan probe. Lanes: M, 100 bp ladder; 1-8, eight $S$. edentatus adult worms; (9) PCR negative control; Lanes 10-13 show adult cyathostomin DNA amplified with the CY1 and CY18 combination of primers; $10, C$. longibursatus; $11, C$. nassatus; 12, C. goldi; 13, C. ashworthi; D, DIG marker.

Southern blot hybridisation of the probes designed to detect $C$. longibursatus, $C$. goldi, $C$. nassatus and $C$. ashworthi individuals (Fig. 2) showed $100 \%$ species-specificity. For example, the Southern blot using the C. longibursatus probe is shown in its entirety (Fig. 2B). It is clear that this probe only hybridised to DNA amplified from the homologous species. No hybridisation was detected against products amplified from the other 15 species. The Pan probe hybridised to all products derived from all cyathostomin species (Fig. 2C). The lack of hybridisation observed to one individual from group 6 and group 8 (Fig. 2C) reflected the poor amplification of IGS products from these individual worms. Despite this, the $C$. longibursatus probe still hybridised to amplified product from this individual worm (Fig. 2B, group 8) emphasising the sensitivity of this system. However, in the case of the $C$. ashworthi individual, there was no hybridisation with the homologous probe (Fig. 2F, group 6). Probes designed to detect $C$. nassatus and $C$. goldi hybridised to each of the three individuals tested from the homologous species (Figs. $2 \mathrm{D}$ and $2 \mathrm{E}$, respectively) but not to any DNA amplified from adults worms of the other 15 species (not shown). Despite the fact that the CY1 and CY18 primers amplified DNA from the $S$. edentatus individuals, these products did not hybridise with the Pan probe (Fig. 4).

A molecular approach to species identification is important for investigating the basic biology of small strongyles, however, there is no current diagnostic method for identifi- cation of species at the egg and larval stages. In order to investigate if this system has potential to detect the species of cyathostomins at these stages, experiments were initiated using PCR products amplified from individual representative eggs, L3 and L4. Fig. 5 shows DNA amplified from four individual eggs and also DNA amplified from a pool of five and ten eggs using the CY1 and CY18 primers. The Pan probe hybridised to product in all samples (Fig. 5B). The probe for $C$. longibursatus species hybridised to product amplified from each of the four individual eggs (Fig 5C). The probes for C. ashworthi, C. goldi and C. nassatus did not hybridise to these products (not shown). In the case where DNA was amplified from five eggs together, only the probe designed from $C$. goldi sequence hybridised (Fig. 5D) and where product from ten eggs was tested, both the $C$. goldi and $C$. longibursatus probes hybridised. DNA was amplified from nine individual L3 (Fig. 6A) and tested with the Pan probe and the four species probes used above (Fig. 6). The Pan probe bound to DNA amplified from all individuals (Fig. 6B), whilst the C. longibursatus probe hybridised to five products from these L3 (Fig. 6C) and the C. goldi probe to DNA generated from one individual L3 (Fig. 6D). The other two probes did not hybridise to any DNA amplified from these L3 (not shown). Finally, DNA was amplified from eight individual L4 (Fig. 7A). Again, the Pan probe hybridised to all products generated from the $\mathrm{L} 4$ stages (Fig. 7B). In contrast to the results with products

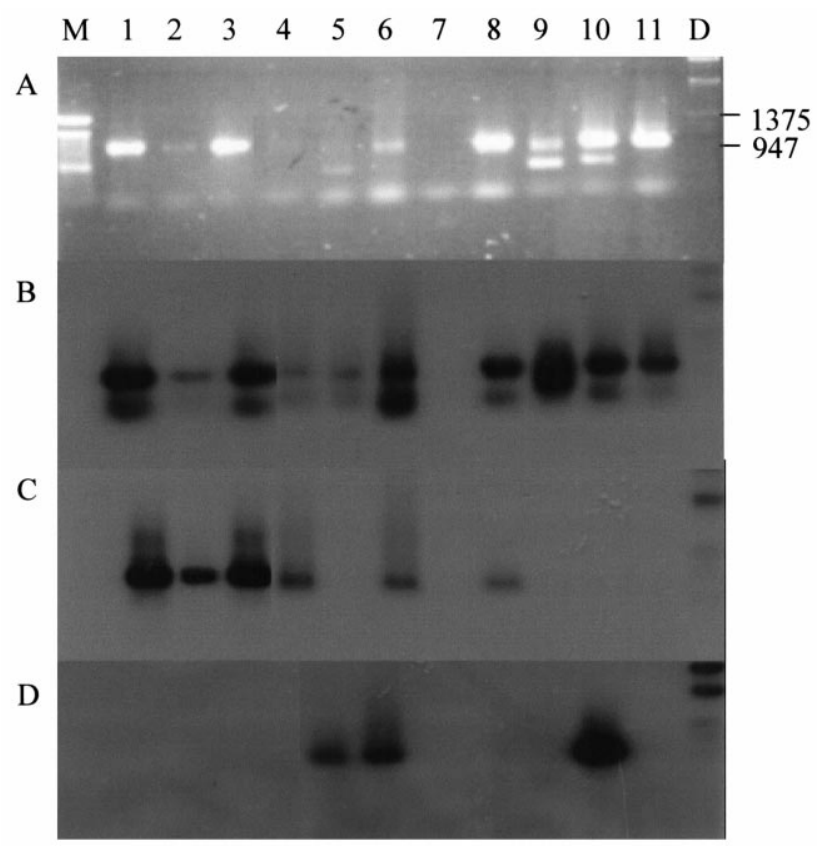

Fig. 5. (A) Electrophoresis in a $1 \%$ agarose gel of the PCR products amplified directly from cyathostomin eggs using primers CY1 and CY 18. Lanes: M, 100 bp ladder; 1-4, single eggs; 5 , five eggs; 6 , ten eggs; 7, PCR negative control; 8, C. longibursatus adult; 9, C. nassatus adult; 10, C. goldi adult; 11, C. ashworthi adult; D, DIG marker. (B) Southern blot of gel in A hybridised with Pan probe. (C) Southern blot of gel in A hybridised with $C$. longibursatus probe. (D) Southern blot of gel in A hybridised with C. goldi probe 


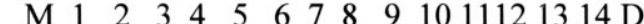

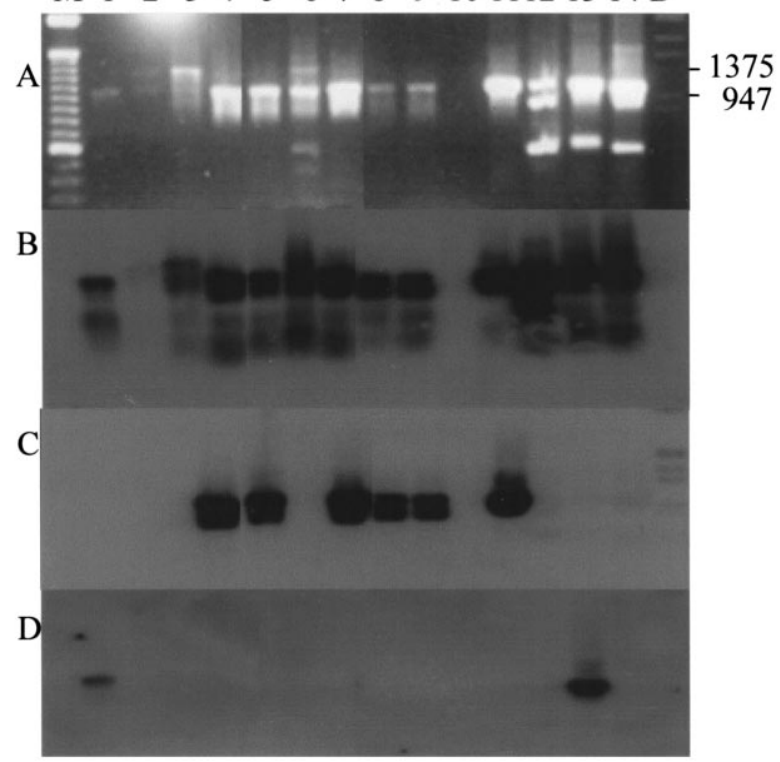

Fig. 6. Electrophoresis in a $1 \%$ agarose gel of the PCR products amplified directly from exsheathed infective L3 stage cyathostomins using primers CY1 and CY 18. Lanes: M, 100 bp ladder; 1-9, single L3; 10, PCR negative control; 11, C. longibursatus adult; 12, C. nassatus adult; 13, C. goldi adult; 14, C. ashworthi adult; D, DIG marker. (B) Southern blot of gel in A hybridised with Pan probe. (C) Southern blot of gel in A hybridised with C. longibursatus probe. (D) Southern blot of gel in A hybridised with $C$. goldi probe.

amplified from eggs and L3, the C. nassatus probe hybridised to four of these eight L4 (Fig. 7C). Neither the C. goldi nor the $C$. longibursatus probes hybridised to any of these products (not shown). In all cases the species-specificity of the probes was demonstrated by the inclusion of PCR product from a morphologically identified adult of the homologous species.

This study reports the use of four oligoprobes designed from short sequences within the IGS region that allow identification of individual cyathostomin species at the adult, egg, L3 and L4 stages. The current method was chosen over this and other commonly used techniques, such as RFLPs (Gasser et al., 1996), for several reasons. Firstly, the amplification of several PCR products from the IGS region of individual worms (see Fig. 2) would make it very difficult to analyse the products of restriction enzyme digestion. Secondly, we aim to adapt this method to a higher throughput PCR-ELISA technique for the rapid analysis of large numbers of eggs, L3 and L4, and the probes shown here are applicable to this type of analysis. Investigation of these small stages with several probes without prior DNA extraction determines that our system must be PCR-based. For the majority of amplifications, the size of the product was consistent across all three individuals representing a species, with the exception of $C$. calicatus, $C$. bidentatus and C. leptostomum. This may reflect greater intraspecific variability in sequence for these species. Alternatively, the size variation may represent the presence of multiple tandem repeat sequences within the amplified products. Species-specific probes selected for Southern blot analysis were restricted in size due to the high level of conservation in the IGS region, which dictated use of low hybridisation temperatures. However, under these conditions, non-specific binding was not a problem. Furthermore, in some cases these short DNA sequences were specific when there were only two bp mismatches. For example, the probe designed from the C. ashworthi sequence had only two bp differences from the sequence showing highest similarity $(C$. leptostomum). A 3-bp difference was selected for the $C$. goldi probe when compared with those species showing greatest similarity across the variable region of the IGS, whilst the $C$. longibursatus probe was designed from a more speciesspecific region. All four probes were shown to be highly specific, furthermore when these probes were tested against nine $(C$. longibursatus and $C$. goldi) or ten $(C$. ashworthi and $C$. nassatus) more individual worms, positive hybridisation was demonstrated in all cases (not shown). The sequence of the fifth probe, the Pan probe, was selected due to its $100 \%$ identity in a larger region of the IGS sequenced previously in five cyathostomin species (Kaye et al., 1998). This probe bound to the IGS PCR products of all cyathostomin species tested, but not to DNA amplified from $S$. edentatus. This supports the use of this probe for identification of members of this tribe of nematodes. The hybridisation of the Pan probe to several products amplified from individual worms of some species reflects that the

\section{$\begin{array}{llllllllllllllll}M & 1 & 2 & 3 & 4 & 5 & 6 & 7 & 8 & 9 & 10 & 11 & 12 & 13 & \mathrm{D}\end{array}$}

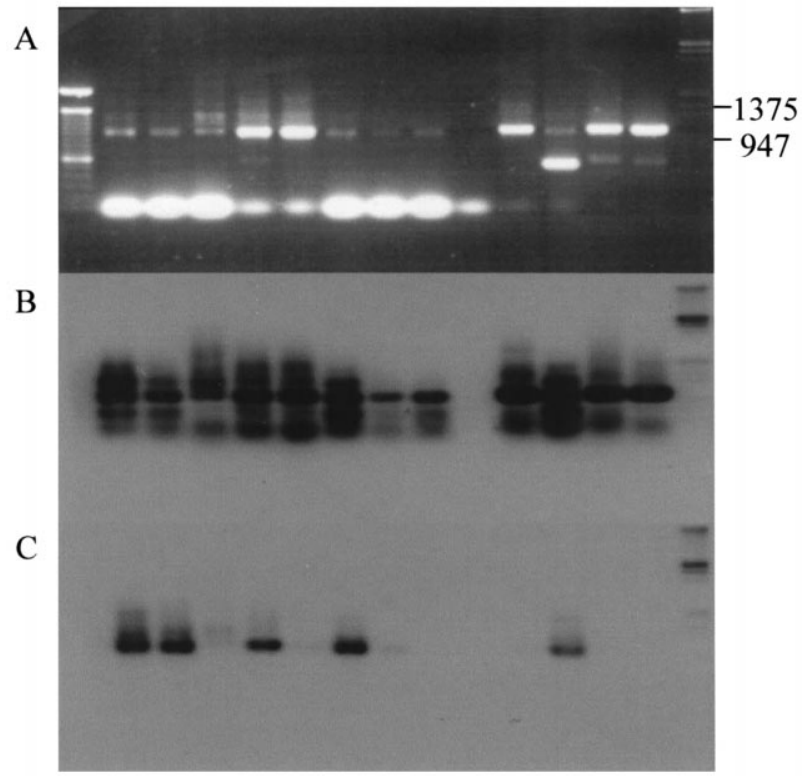

Fig. 7. (A) Electrophoresis in a $1 \%$ agarose gel of the PCR products amplified from genomic DNA extracted from L4 stage cyathostomins using primers CY1 and CY 18. Lanes: M, 100 bp ladder; 1-8, single L4; 9, PCR negative control; 10, C. longibursatus adult; 11, C. nassatus adult; 12, C. goldi adult; 13, C. ashworthi adult; D, DIG marker. (B) Southern blot of gel in A hybridised with Pan probe. (C) Southern blot of gel in A hybridised with $C$. nassatus probe. 
region from which it was designed is external to the region containing the tandem repeats. Based on sequencing of several products of different sizes from $C$. catinatum individuals, these repeats appear to be responsible for the different size products amplified from individuals of some species. Tandem repeats in this region have been described in other nematode species (Vahidi and Honda, 1991).

Some 51 species of cyathostomins have been recorded in horses, donkeys and/or zebra worldwide (Lichtenfels et al., 1998), but 10 of these have been reported only from zebra or donkeys and a few are known to have a very limited distribution. We have determined the specificity of these probes for 16 of the 40 species that occur in horses. In order to increase the repertoire of cyathostomin species available for analyses, adult cyathostomins are routinely collected from horses at a local abattoir and identified. However, the 16 species represented in this analysis include the most common species with the greatest intensity of infection (Table 1; Ogbourne, 1976; Bucknell et al., 1995; Lyons et al., 1997) and all of those species identified so far which show greatest sequence similarity to the probes being tested. C. catinatum has been reported as a particularly prevalent species (Bucknell et al., 1995) and we are currently developing a probe for its identification. In addition to the members of the Cyathostominae, samples may contain parasites of other genera. However, we have shown that DNA did not amplify with the CY1 and CY18 primers using $P$. equorum and $O$. equi templates, whilst products amplified from large strongyle individuals did not hybridise with the Pan probe.

Preliminary studies also showed that these small oligoprobes could identify individual species from within a mixed population of eggs and, moreover, indicate the species present in individual egg, L3 and L4 samples. The eggs and L3 tested here were harvested from the same population of benzimidazole resistant worms from a group of grazing horses, whilst the L4 analysed were obtained from faeces from horses located in a different part of the UK. These results emphasise the utilisation of this PCR-based technique for use in non-invasive epidemiological, prevalence and pathogenesis studies at the single species level. As mentioned above we hope to further adapt the use of these probes to a PCR-ELISA format to allow a sensitive, highthroughput analysis of numerous samples of all developmental stages. This is currently under evaluation in our laboratory, in combination with the design and validation of new probes for other common cyathostome species.

\section{Acknowledgements}

We would like to acknowledge the Horserace Betting Levy Board for generously funding this project, Professor Andy Tait for use of his laboratories in the Department of Veterinary Parasitology, University of Glasgow and James McGoldrick, for his invaluable help. We would like to thank
Dr John Cox, Miss Sam Dowdall and Mr Jason Brown, University of Liverpool, for provision of some of the samples used in this study. J.B.M. is funded by the International League for the Protection of Horses.

\section{References}

Beelitz, P., Gothe, R., 1997. Spectrum of species and incidence of endoparasites in yearlings and adult horses from breeding farms with regular anthelmintic prophylaxis lasting for years in Upper Bavaria. Tierarztliche Praxis 25, 445-50.

Bucknell, D.G., Gasser, R.B., Beveridge, I., 1995. The prevalence and epidemiology of gastrointestinal parasites of horses in Victoria. Australia. Int. J. Parasitol. 25, 711-24.

Gasser, R.B., Stevenson, L.A., Chilton, N.B., Nansen, P., Bucknell, D.G., Beveridge, I., 1996. Species markers for equine Strongyles detected in intergenic rDNA by PCR RFLP. Mol. Cell. Probes 10, 371-8.

Giles, C.J., Urquhart, K.A., Longstaffe, J.A., 1985. Larval cyathostomiasis (immature trichoneme induced enteropathy), a report of 15 clinical cases. Equine Vet. J. 17, 196-201.

Jorgensen, R., 1975. Isolation of infective Dictyocaulus larvae from herbage. Vet. Parasitol. 1, 61.

Kaye, J.N., Love, S., Lichtenfels, J.R., McKeand, J.B., 1998. Comparative sequence analysis of the intergenic spacer region of cyathostome species. Int. J. Parasitol. 28, 831-6.

Klei, T.R., French, D.D., 1998. Special workshop presentation-small strongyles; an emerging parasite problem for horses. Equine Pract. 20, 23-26.

Langrova, I., 1998. Seasonal prevalence and intensity of faecal helminth (larval) output in various categories of herds of horses during two grazing seasons. Helminthologia 35, 43-50.

Lichtenfels, J.R., Kharchenko, V.A., Krecek, R.C., Gibbons, L.M., 1998. An annotated checklist by genus and species of 93 species level names for 51 recognised species of small strongyle (Nematoda: Strongyloidea: Cyathostminea) of horses, asses and zebras of the world. Vet. Parasitol. 79, 65-79.

Love, S., McKeand, J.B., 1997. Cyathostomosis: practical issues of treatment and control. Equine Vet. Education 9, 253-6.

Love, S., Escala, J., Duncan, J.L., MacLean, J.M., 1992a. Studies on the pathogenic effects of experimental cyathostome infections in ponies. Proceedings VIth Conference Equine Infectious Diseases 22, 149-55.

Love, S., Mair, T.S., Hillyer, M.H., 1992b. Chronic diarrhoea in adult horses: a review of 51 referred cases. Vet. Rec. 130, 217-9.

Lyons, E.T., Tolliver, S.C., Collins, S.S., Drudge, J.H., Granstrom, D.E., 1997. Transmission of some species of internal parasites in horses born in 1993, 1994 and 1995 on the same pasture on a farm in central Kentucky. Vet. Parasitol. 70, 225-40.

Matthews, A.G., Morris, J.R., 1995. Cyathostomiasis in horses. Vet. Rec. $136,52$.

Murphy, D., Love, S., 1997. The pathogenic effects of experimental cyathostome infections in ponies. Vet. Parasitol. 70, 99-110.

Ogbourne, C.P., 1976. The prevalence, relative abundance and site distribution of nematodes of the subfamily Cyathostominae in horses killed in Britain. J. Helminthol. 50, 203-14.

Reinemyer, C.R., Smith, S.A., Gabel, A.A., Herd, R.P., 1986. The prevalence and intensity of internal parasites of horses in the USA. Vet. Parasitol. 5, 75-83.

Torbert, B.J., Klei, T.R., Lichtenfels, J.R., Chapman, M.R., 1986. A survey in Louisiana of intestinal helminths of ponies with little exposure to anthelmintics. J. Parasitol. 72, 926-30.

Vahidi, H., Honda, B.M., 1991. Repeats and subrepeats in the intergenic spacer of rDNA from the nematode Meloidogyne arenaria. Mol. Gen. Genet. 227, 334-6.

Vanloon, G., Deprez, P., Muylle, E., Sustronk, B., 1995. Larval cyathostomiasis as a cause of death in two regularly dewormed horses. J. Vet. Med. 42, 301-6. 